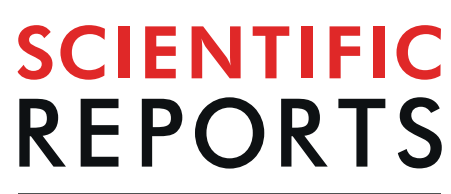

natureresearch

\title{
CRISPR/Transposon gene integration (CRITGI) can manage gene expression in a retrotransposon-dependent manner
}

\begin{abstract}
Miki Hanasaki \& Hiroshi Masumoto $\mathbb{B}^{*}$
The fine-tuning of gene expression contributes to both basic science and applications. Here, we develop a novel gene expression technology termed CRITGI (CRISPR/Iransposon gene integration). CRITGI uses CRISPR/Cas9 to integrate multiple copies of the plasmid pTy1 into Ty1 loci, budding yeast retrotransposons. The $\mathrm{p}$ Ty1 plasmid harbors a Ty1 consensus sequence for integration, a gene of interest with its own promoter and a selection marker gene. Interestingly, the expression of the pTy 1 gene in Ty 1 loci could be induced in synthetic complete amino acid depletion medium, which could activate the selection marker gene on pTy1. The induction or repression of the gene on pTy1 depended on Ty1 transcription. Activation of the selection marker gene on pTy1 triggered Ty1 transcription, which led to induction of the gene on pTy1. The gene on pTy1 was not transcribed with Ty1 mRNA; the transcription required its own promoter. Furthermore, the trimethylation of histone $\mathrm{H} 3$ on lysine 4 , a landmark of transcriptionally active chromatin, accumulated at the $5^{\prime}$ end of the gene on pTy 1 following selection marker gene activation. ThUs, CRITGI is a unique gene regulation system to induce the genes on pTy1 in amino acid depletion medium and utilizes Ty1 transcription to create a chromatin environment favorable for the transcription of the genes on pTy1.
\end{abstract}

Clustered regularly interspaced palindromic repeats (CRISPR) have evolved as a primitive immune system in prokaryotes with the ability to precisely target and edit any genome $\mathrm{e}^{1-4}$. The Cas9 endonuclease of the Class II CRISPR system initially binds to a single-stranded RNA (single guide (sg) RNA) containing a short stretch of RNA ( 20 bases) that binds and recruits the Cas9/RNA complex to the corresponding sequence within a target genome locus that is also marked with a protospacer adjacent motif (PAM) $5^{\prime}$-NGG-3' sequence ${ }^{5,6}$. The Cas9/ sgRNA complex causes a double-stranded break (DSB) at the position upstream of the PAM. DSBs introduced at the genome position(s) by the Cas9/sgRNA complex have enabled gene replacement, gene deletions and single base editing in many eukaryotic organisms, including humans ${ }^{7-11}$. Saccharomyces cerevisiae, or budding yeast, is one of the most studied and genetically and biologically tractable organisms. The tractability of yeast in both basic science and industry stems from the ability to rapidly edit and manipulate its genome. Together with the evolved CRISPR system suitable for budding yeast cells, various efforts have provided a new suite of molecular tools using the CRISPR system that have been applied to a diverse array of methodologies in budding yeast, including multiplexed editing, chromosome splitting, transcriptional modulation, synthetic genome engineering and gene drive technology ${ }^{12-17}$.

\section{Results and Discussion}

In this research, our first goal was to develop a novel gene expression tool using the CRISPR/Cas9 system suitable for budding yeast. To obtain a strain with stable expression of the protein of interest, we usually select a strategy to integrate a linearized expression vector into the target locus in the chromosome by homologous recombination $(\mathrm{HR})^{18}$. Multiple integrations of an expression vector are expected to increase the expression of the protein 
a

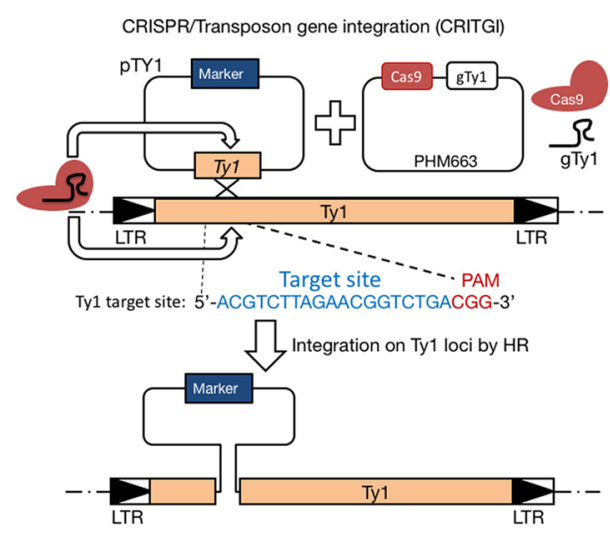

b

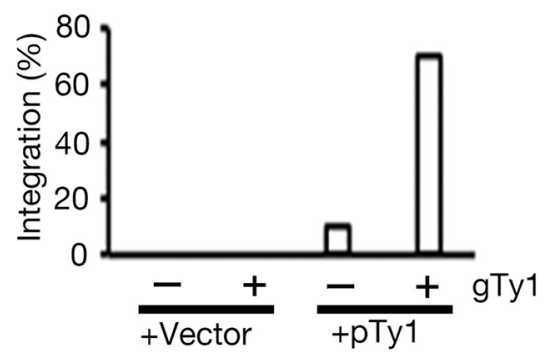

C
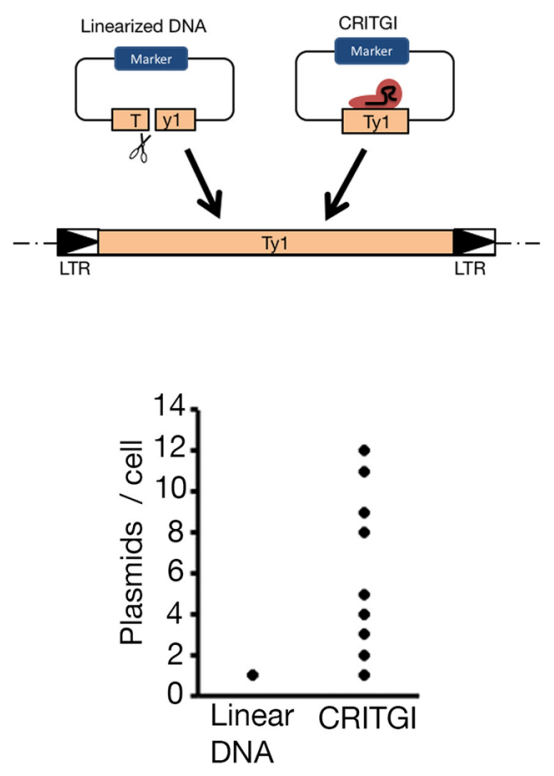

Figure 1. CRISPR/Transposon gene integration (CRITGI) can introduce multiple plasmids into Ty1 loci. (a) A diagram of CRITGI. Two types of plasmids (pTy1 and PHM663) were simultaneously transformed into yeast. Blue colored bases and red colored bases indicate the target sequence and 5' PAM sequence of gTyl, respectively. Marker: marker gene for selection of transformants. (b) Percentage of correct integration of the pTy1 plasmid into Ty1 loci. YIplac128 was used as a vector. (c) Comparison of the integrated pTy1 plasmid number in Tyl loci between the linear plasmid and CRITGI. The Sal I-digested linear pTyl plasmid (linear DNA) and the mixture with the PTY1 and PHM663 plasmids (CRITGI) were transformed into yeast cells. The number of integrated pTY1 plasmids on Ty1 loci was calculated for the ACT1 gene as one copy gene per genome by using real time (RT)-PCR, which included transformants derived from linear DNA $(n=8)$ and those from CRITGI $(\mathrm{n}=20)$.

of interest. However, we usually obtain a strain harboring one copy of a plasmid in a chromosome, even though many target loci exist in the chromosome. To establish a method for multiple integrations of an expression vector, we tested the CRISPR/Cas9 system to introduce multiple copies of a plasmid into multiple target loci in the yeast genome. We used Ty1, one of five yeast retrotransposons (Ty1 to Ty5), as which is located at multiple sites throughout the genome; 31 are complete forms, and truncated forms occupy $3 \%$ of the total genome volume ${ }^{19,20}$. The coding region of Ty1 is flanked by two long terminal repeats (LTRs) that function as the transcriptional promoter and terminator $\left(\right.$ reviewed $\mathrm{in}^{20}$ ). Shi and colleagues have already developed Di-CRISPR to integrate multiple copies of gene clusters into the LTR regions of Ty1 and Ty2 (delta) sites ${ }^{12}$. We used the sequence region from 1682 bp to 2518 bp (836 bp length) of YPRWRTy1-3, named the Ty1 HR sequence, as a vector integration site, which is highly conserved among the $31 \mathrm{Ty} 1$ complete forms ${ }^{21}$. A mixture of the integration vector harboring the Ty1 HR sequence (pTy1) and PHM663 plasmid expressing Cas9 and sgRNA to recognize the Ty1 HR sequence (gTy1) was transformed into yeast cells. The Cas9/gTy1 complex can introduce DSBs within the Ty1 HR sequence in the pTy1 plasmid and then integrate the plasmid into the Ty1 locus by HR (Fig. 1a). We named this plasmid integra-

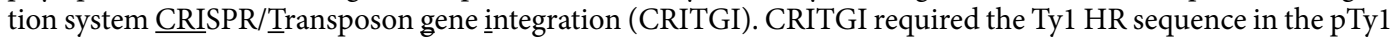
plasmid to obtain transformants; the transformation efficiency of the pTyl plasmid significantly dropped without the Ty1 HR sequence (Fig. S1). gTY1 sgRNA was required for pTyl plasmid integration into Tyl loci with 75\% accuracy, in contrast to $10 \%$ accuracy without gTy1 (Fig. 1b). Furthermore, direct sequencing revealed that the sequence at the pTy1 plasmid-integration site on the Ty1 locus in all transformants $(n=10)$ was identical to the reference sequence of the parent strain (BY4742) (Fig. S2), suggesting that the pTy1 plasmid was correctly integrated into the Ty1 locus by HR. Multiple DSBs by Cas9/gTY1 in Ty1 loci might trigger rearrangement among chromosomes. Pulse field gel electrophoresis (PFGE) showed that the size of any chromosome band had no obvious change in transformants compared to wild type (Fig. S3), suggesting that CRITGI could integrate the pTy1 plasmid without large-scale genomic rearrangement. Next, we used quantitative PCR (qPCR) to count the copy number of pTy1 plasmids in the transformants. Although transformation using linearized pTy1 plasmid resulted in a strain with a single copy of the pTyl plasmid in the Tyl locus (Fig. 1c; Linear DNA), CRITGI could 
introduce multiple copies of pTy1 plasmids into Ty1 loci (Fig. 1c: CRITGI). Thus, CRITGI enables multiple copy numbers of pTy1 plasmids to integrate into Ty1 loci, as in Di-CRISPR ${ }^{12}$. Furthermore, we examined whether CRITGI simultaneously enabled several different types of pTy1 plasmids ( 3 species) to integrate into Ty1 loci. We obtained transformants with several species of pTyl plasmids, in which the frequency of plasmid combination was reduced to $50 \%$ (2 pair) and $10 \%$ ( 3 pair) among the total transformants examined $(n=10)$ (Fig. S4D). Thus, CRITGI is able to introduce not only multiple copies of pTy1 plasmids but also various species of pTy1 plasmids into Ty1 loci.

Several studies have claimed that the gene inserted in the Tyl element may undergo transcriptional cosuppression, which is defined as high gene copy number-triggered homology-dependent gene silencing ${ }^{22,23}$. Therefore, we examined whether a gene in the pTy1 plasmid within Ty1 loci was able to express or not. The pTy1-H3 plasmid harbors the HHT1 gene encoding the histone $\mathrm{H} 3$ protein with a FLAG epitope tag at the N-terminus driven by the strong constitutive TDH3 promoter (Fig. 2a: top) ${ }^{24}$. An immunoblot using an $\alpha$-FLAG antibody showed that either a faint or undetectable level of FLAG-H3 was expressed in cells with various copies of pTy1-H3 plasmid grown in yeast extract-peptone-dextrose (YPD) medium, a conventional rich medium (Fig. S5A,B). These results suggest that FLAG-H3 expression is downregulated within the Tyl element. The pTy1-H3 plasmid harbors the HIS3 gene as a marker gene, which is activated in cells grown in synthetic complete histidine dropout medium (SC-His). We hypothesized that activation of the HIS3 gene might induce Flag-HHT1 gene transcription from the pTy1-H3 plasmid. To test this hypothesis, we examined the expression of the FLAG-HHT1 gene in cells with pTy1-H3 grown in SC-His medium. Surprisingly, FLAG-H3 was detected in cells with pTy1-H3 grown in SC-His but not in YPD (Fig. 2a (CRITGI); lanes 1 and 2), although FLAG-H3 expression was detected in both SC-Ura (uracil dropout) and YPD using cells with the FLAG-HHT1 gene and the TDH3 promoter integrated in the URA3 locus (Fig. 2a (URA3); lanes 3 and 4). Thus, marker gene activation triggers the expression of the gene in cells with the pTy1 plasmid, and this transcriptional repression correlates with the Ty1 element. FLAG-H3 expression in cells with pTy1-H3 was induced in SC-His only, but not in SC-Met (methionine dropout) or SC (complete set of amino acids essential for budding yeast) (Fig. S6). This means that the gene expression accompanying marker activation is severely restricted to the kind of amino acid. We next examined whether the protein expression level was proportional to the integration copy number of the pTy1 plasmid. The pTy1-V plasmid can express Venus, a variant yellow fluorescent protein (YFP), with a FLAG epitope tag at the N-terminus under a synthetic promoter $(P s y n)$ and synthetic terminator (Tguo1) (Fig. 2b: top) ${ }^{25-27}$. In cells with the pTy1-V plasmid grown in SC-Leu (leucine dropout), FLAG-Venus was rarely detected in cells with a single copy of the pTy1-V plasmid (Fig. 2b bottom: lane 1). In cells with 3 copies of the plasmid, a faint band of FLAG-Venus was detected, and the signal intensity of the Flag-Venus band reached a plateau in cells with both 6 and 13 copies of the pTy1-V plasmid, although FLAG-Venus was rarely detected in cells with 13 copies of pTy1-V plasmids grown in YPD (Fig. $2 \mathrm{~b}$ bottom: lane 2 to 5). Thus, protein expression levels are proportional to the copy number of pTyl plasmids. Next, we examined whether the gene transcription and protein expression from the plasmid were linked to Tyl transcription. Cells with pTy1-V plasmids (10 copies) were cultured in YPD and then released into SC-Leu. Real-time quantitative PCR (RT-qPCR) was used to monitor the mRNA levels of Ty1, FLAG-Venus, and LEU2 (ACT1 as a reference). Ty1, LEU2 and Venus mRNA levels simultaneously increased after cells were released into SC-Leu (Fig. S7A-C). Similar to the fluctuation of Venus mRNA, an immunoblot using an $\alpha$-FLAG antibody detected the FLAG-Venus protein from $2 \mathrm{~h}$ after the cells were released into SC-Leu (Fig. 2c: lane 1 to 4 ). Thus, FLAG-Venus expression correlated with an increase in Ty1 mRNA together with LEU2 gene induction. Next, cells with the pTy1-V plasmid were cultured in SC-Leu and then released into YPD. The mRNA levels of Ty1, LEU2 and Venus simultaneously decreased after being released into YPD medium (Fig. S7D-F). The FLAG-Venus protein was also abolished during the time course, but its disappearance was delayed compared to FLAG-Venus mRNA (Fig. 3d; lanes 1-4 and $\mathrm{S7F}$ ), which is due to the robustness of the Venus protein ${ }^{28}$. Thus, the repression of the transcription of the Venus gene was also linked to the reduction in Ty1 mRNA, together with the inactivation of the LEU2 gene. Altogether, gene expression in the pTyl plasmid is connected by Ty1 mRNA transcription.

Changes in Ty1 transcriptional factors might influence gene transcription in cells with the pTyl plasmid. The SAGA complex, a general transcriptional activator, the Swi/Snf and ISWI chromatin remodeling complexes, and various other transcriptional regulators are involved in Ty1 transcription ${ }^{20,29-34}$ (Fig. 2f). Gcn5 is a general transcriptional activator, and the deletion of $g c n 5$ reduces the transcriptional activities of both LTRs of the Ty1 elements and Psyn promoter ${ }^{20,26}$. The FLAG-Venus protein was rarely detected in the gcn5 $\Delta$ background during the time course (Fig. 2f; lane 4-6). Gcn4 also regulates Ty1 transcription and binds five potential Gcn4-binding motifs $\left(5^{\prime}\right.$-TGAATG- $3^{\prime}$ ) in the vicinity of the LTR region of the Tyl element (Fig. 2f). However, the Tyl mRNA level in $g c n 4 \Delta$ cells remains at almost the same level as wild-type cells ${ }^{34}$. In contrast to the reduction in the FLAG-Venus protein in $g c n 5 \Delta$ cells (Fig. 2f; lane 4-6), the FLAG-Venus protein was constantly expressed in $g c n 4 \Delta$ cells during the time course (Fig. 2 f; lane 1-3). These results suggest that Ty1 mRNA transcription controls gene expression in the pTyl plasmid within the Tyl element.

Jiang reported that transcription of the in-frame Tyl-gene fusion depends on the LTR in the Tyl element ${ }^{23}$. The FLAG-HHT1 gene is in-frame with Ty1 transcription in cells with the pTy1-H3 plasmid. If FLAG-HHT1 mRNA occupied a part of the Ty1 mRNA, FLAG-HHT1 could be expressed without its own promoter (i.e., the TDH3 promoter in the pTy1-H3 plasmid). We tested Flag-H3 expression in both cells with the pTy1-H3 plasmid without the TDH3 promoter region ( $\mathrm{pTy} 1-\mathrm{H} 3 \Delta \mathrm{p}$ ) and cells with the $\mathrm{pTy} 1-\mathrm{H} 3$ plasmid following the marker gene induction (Fig. 3a: top). FLAG-H3 was rarely detected in cells with the pTy1-H3 $\Delta$ p plasmid regardless of the integration copy number of the pTy1-H3 $\Delta$ p plasmid, although FLAG-H3 was detected in cells with pTy1-H3 (Fig. 3a: bottom). This suggests that the expression of FLAG-H3 in the pTy1-H3 plasmid needs its own promoter within the Tyl element. From this result, we hypothesized that marker gene induction could "open" the Tyl chromatin structure, which is favorable for gene transcription within the pTyl plasmid. To demonstrate this hypothesis, we performed Micrococcus nuclease (MNase)-chromatin immunoprecipitation (ChIP) to examine the level of 
a
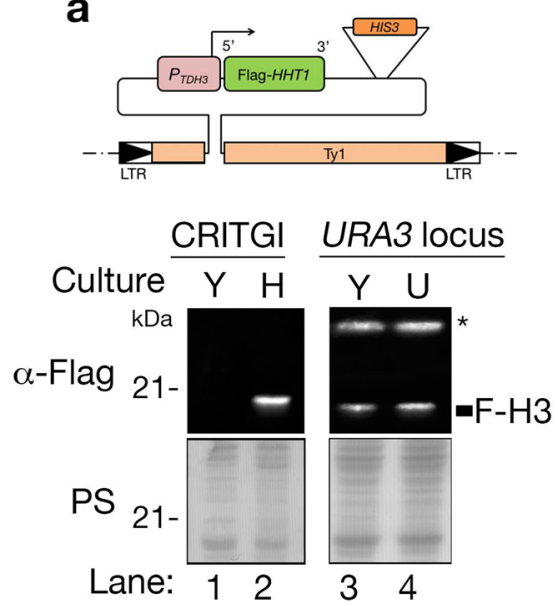

C

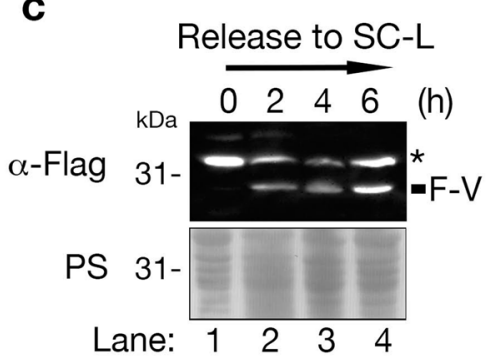

e

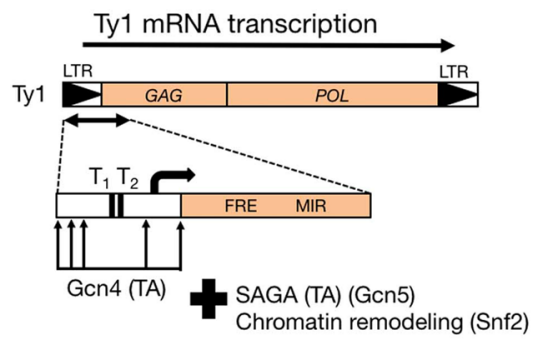

b
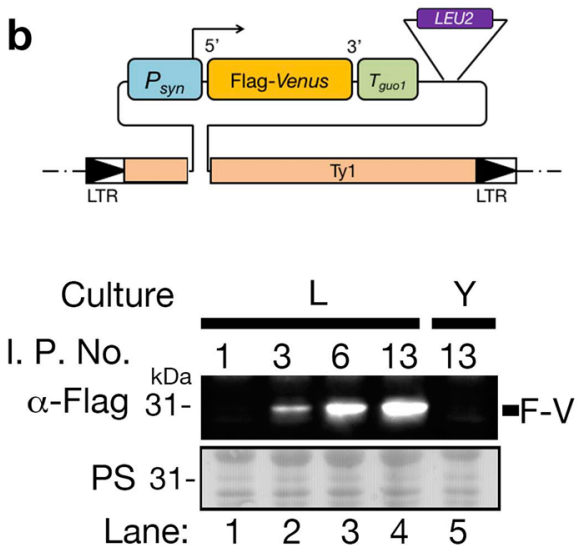

d

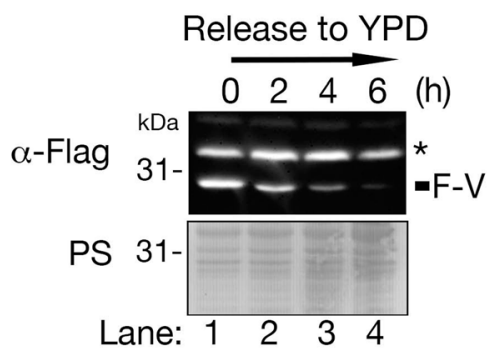

f

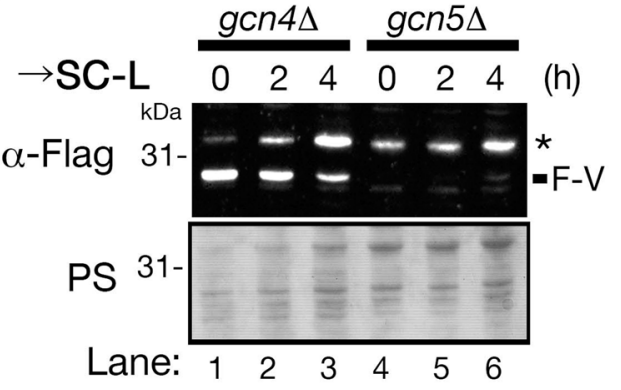

Figure 2. CRITGI regulates protein expression in a Ty1-dependent manner. (a) Flag-H3 expression in cells with the pTy1-H3 plasmid was induced in medium to activate the marker gene. A schematic representation of the pTy1-H3 plasmid integrated into the Ty1 locus (top). The FLAG-HHT1 gene encodes Flag-histone H3 (F-H3). $P_{T D H 3}$ : TDH3 promoter. HIS3: HIS3 marker gene. Immunoblotting using an anti-Flag antibody detected Flag-H3 in cell extracts (bottom). Cells were cultured in either synthetic complete (SC) histidine dropout media (SC-His) or YPD media at $25^{\circ} \mathrm{C}$ overnight. CRITGI: HMY1466 strain (wild-type cells with the pTy1-H3 plasmid (I. P. No. = 11)). URA3 locus: HMY1502 strain harboring a single copy of Flag-histone $\mathrm{H} 3$ with the TDH3 promoter in the URA3 locus. I. P. No.: integrated plasmid number. Y: YPD culture. H: SC-His culture. U: SC-Ura culture. PS: Ponceau S staining. *Nonspecific protein band. Cell extracts were analyzed by immunoblotting using $\alpha$-Flag antibody. (b) The expression level of Flag-Venus increased proportionally with the integrated plasmid number in Ty1 loci. A schematic representation of the pTy1-V plasmid integrated into the Ty1 locus (top). The pTy1-V plasmid harbors the Flag-Venus gene with the Psyn promoter, Tguo1 terminator, and LEU2 marker gene. Four types of HMY1494 strains (wild-type cells with the pTy1-V plasmid (I. P. No. $=1,3,6$ and 13)) were cultured in YPD at $25^{\circ} \mathrm{C}$ and then released into either SC-L or YPD. L: SC-Leu culture. F-V: Flag-Venus. (c and d) Immunoblot detection of Flag-Venus protein over time. The HMY1476 strain (wild-type cells with the pTy1-V plasmid (I. P. No.=10)) was employed. (c) The HMY1476 strain was cultured in YPD at $25^{\circ} \mathrm{C}$ and then released into SC-L. (d) The HMY1476 strain was cultured in SC-L at $25^{\circ} \mathrm{C}$ and then released into YPD. (e) A schematic representation of Ty1 transcriptional regulation modified from Servant, G. et al., ${ }^{33}$. GAG and $P O L$ are analogs to the retrovirus genes $G A G$ and $P O L$, respectively. T: TATA box sequence. TA: transcriptional activator. (f) Flag-Venus expression was influenced by trans-acting factors for Ty1 transcription. The HMY1491 strain $(g c n 4 \Delta$ with pTy1-V plasmid (I. P. No. $=24))$ and HMY1492 strain $((g c n 4 \Delta$ with pTy1-V plasmid (I. P. No. $=32)$ ) were cultured in YPD at $25^{\circ} \mathrm{C}$ and then released into SC-L $(\rightarrow$ SC-L). 
a
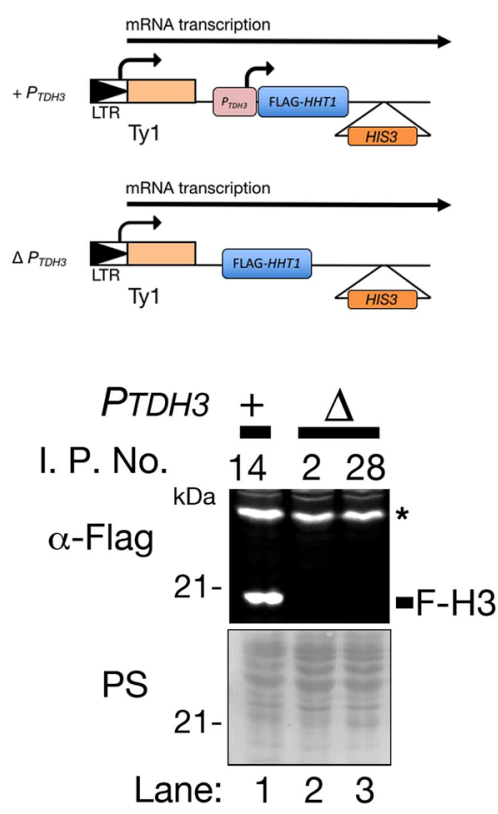

b

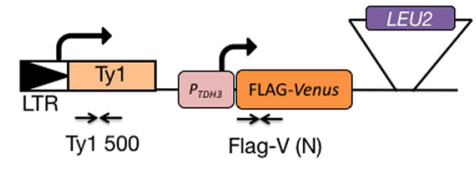

MNase-ChIP ( $\alpha-\mathrm{H} 3-\mathrm{K} 4 \mathrm{me})$

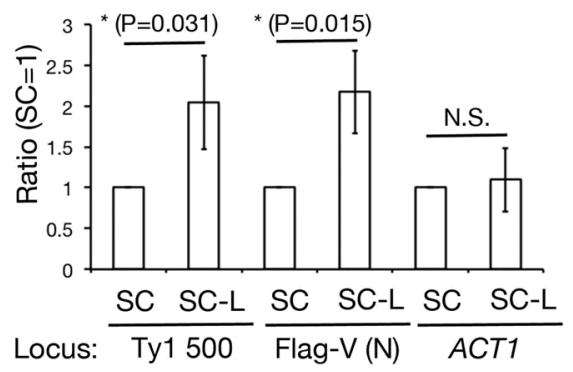

C
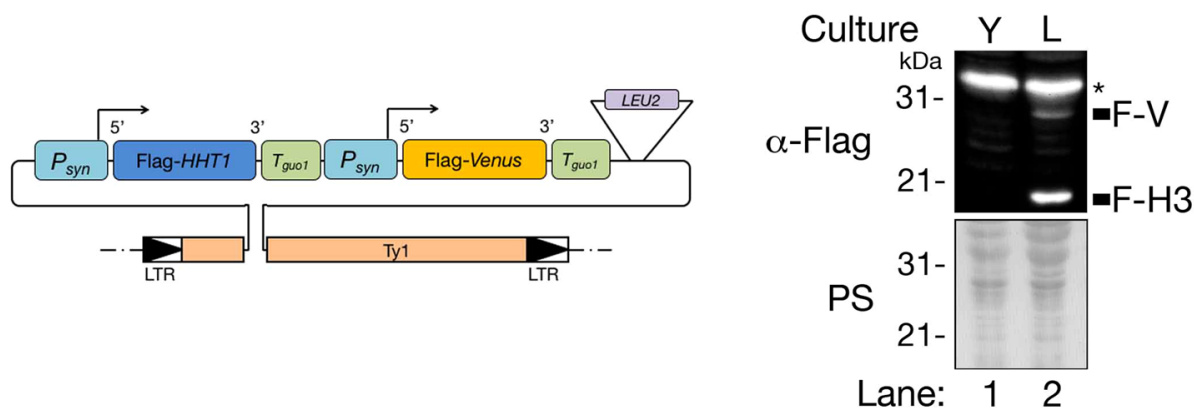

Figure 3. CRITGI promotes a chromatin structure favorable for gene expression. (a) A schematic representation of the FLAG-HHT1 gene integrated into the Ty1 element in the presence and absence of $P_{T D H 3}$ $\left(+P_{T D H 3}\right.$ and $\Delta P_{T D H 3}$, respectively) (top). Immunoblotting using an anti-Flag antibody detects Flag-H3 in cell extracts (bottom). Two types of HMY1496 strains (wild-type cells with the pTy1-H3 $\Delta$ p plasmid (I. P. No. $=2$ and 28, respectively)) and the HMY1466 strain (wild-type cells with pTy1-H3 (I. P. No. = 14)) were cultured in SC-His at $25^{\circ} \mathrm{C}$ overnight. As in Fig. 2, the nitrocellulose membrane, in which proteins had been transferred to, was stained with $0.1 \%$ Ponceau S buffer and scanned to quantify the protein level in each lane. After destaining the membrane by TBS-T, the same membrane was used for the Western blot using the $\alpha$-Flag antibody. (b) MNase-ChIP assay using anti-histone H3-K4me3 antibody. The HMY1476 strain (wild-type cells with the pTy1-V plasmid (I. P. No. =4)) was cultured either in SC or in SC-Leu (SC-L) at $25^{\circ} \mathrm{C}$ for $5 \mathrm{~h}$. Ty 1500 and Flag-V $(\mathrm{N})$ were used as target sites for RT-qPCR analysis. $* P<0.05$. Unpaired t-test (one-tail). Error bars represent the standard deviation of three biological replicates. The ACT1 gene locus was used as a control, and the H3-K4me3 level was not altered between SC and SC-L media. (c) CRITGI can synchronously express two types of genes set in tandem within Tyl loci in SC-Leu, but not in YPD medium. A schematic representation of the pTy1-H3-V plasmid in the Ty1 element. The pTy1-H3-V plasmid harbors two gene sets: the FLAG-HHT1 gene with the Psyn promoter and Tguol terminator, FLAG-Venus gene with the Psyn promoter and Tguo1 terminator in tandem with the LEU2 marker gene (top). The HMY1500 strain (wild-type cells with the pTy1H3-V plasmid (I. P. No. =4)) was cultured either in SC-Leu (L) or YPD (Y) at $25^{\circ} \mathrm{C}$ overnight. Immunoblots detected Flag-histone H3 (F-H3) and Flag-Venus (F-V) in cell extracts (bottom).

trimethylation of histone $\mathrm{H} 3$ on $\mathrm{K} 4(\mathrm{H} 3-\mathrm{K} 4 \mathrm{me} 3)$ in chromatin surrounding the gene on the pTy1-V plasmid ${ }^{35,36}$. Histone $\mathrm{H} 3-\mathrm{K} 4 \mathrm{me} 3$ is a landmark of active gene transcription and is enriched at the $5^{\prime}$ end of the active gene in budding yeast ${ }^{35,36}$. We compared the level of histone $\mathrm{H} 3-\mathrm{K} 4 \mathrm{me} 3$ on the nucleosome both at the start position of Ty1 (Ty1 500) and $5^{\prime}$ end of the Flag-Venus gene (Flag-V (N)) on the pTy1-V plasmid (Fig. 3b; upper model) in cells with pTy1-V plasmids (4 copies) cultured in SC-Leu and SC media. The level of histone H3-K4me3 in both Ty1 500 and Flag-V (N) regions was more significantly increased in the SC-Leu culture than in the SC culture, not in ACT1 region (Fig. 3b). This means that the repressed chromatin within Ty1 became more favorable for transcription following marker gene activation. If the chromatin structure within Tyl was a detrimental factor for controlling gene transcription in the pTy1 plasmid, two different gene units set in tandem in the pTy1 plasmid could be synchronously expressed following marker gene induction. The pTy1-V-H3 plasmid harbors the 


\section{a}

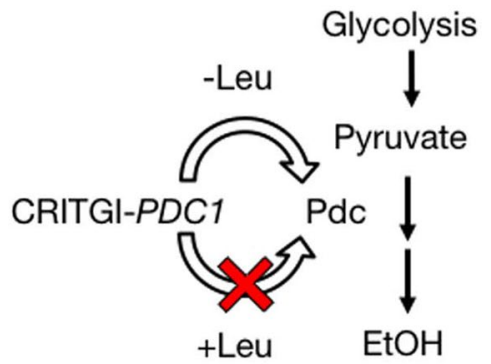

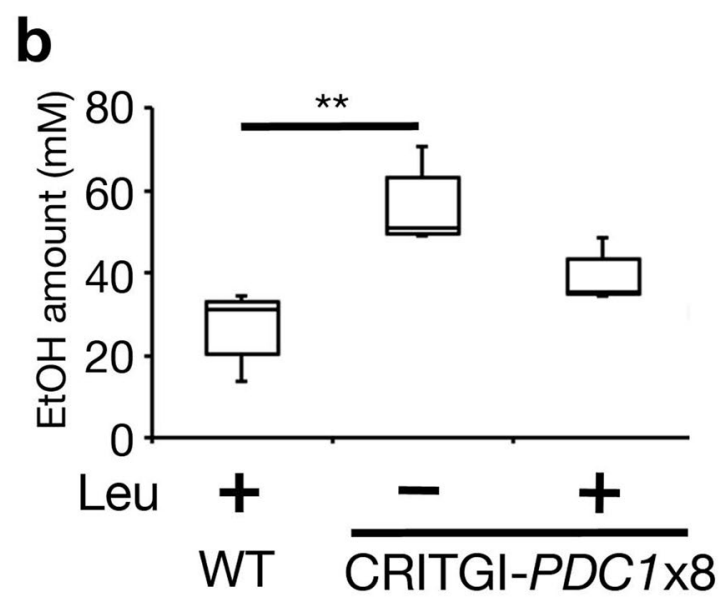

Figure 4. CRITGI can control ethanol production. (a) Pdc1 encoding pyruvate decarboxylase is involved in the conversion of pyruvate to ethanol during alcoholic fermentation. The PDC1 gene is induced in the absence of leucine and repressed in the presence of leucine in cells harboring the pTy1-Pd plasmid containing PDC1 (CRITGI-PDC1). (b) Ethanol production was increased in HMY1504 (wild-type cells with the pTy1-Pd plasmid $\left(\right.$ I. P. No. = 8)) in the presence of Leu. The WT (BY4742) and HMY 1504 strains were cultured at $25^{\circ} \mathrm{C}$ for $24 \mathrm{~h}$ either in SC-Leu ( - Leu) or SC + com $(+$ Leu). $* P<0.05$. Repeated measures ANOVA with Bonferroni correction. Error bars represent the standard deviation of three biological replicates.

following arrayed genes in tandem: the FLAG-Venus gene and FLAG-HHT1 gene with the Psyn promoters and Tguo1 terminators (Fig. 3c: top). Both FLAG-Venus and FLAG-H3 were detected in cells with the pTy1-V-H3 plasmid grown in SC-Leu, although both proteins were rarely detected in YPD (Fig. 3c: bottom). The Flag-Venus level was lower than Flag- H3 (Fig. 3c: bottom) because Flag-H3 can be incorporated into chromatin after the replication fork passes ${ }^{37}$ and the half-life of nucleosomal Flag-H3 extends longer than that of free Flag-Venus (Fig. 2d $)^{38}$. Altogether, marker gene induction can alter the Tyl chromatin environment, making it more favorable for gene transcription from the pTy1 plasmid.

We tested whether CRITGI could be applicable for metabolic engineering. Pyruvate decarboxylase (Pdc) plays an essential role in producing ethanol from pyruvate (Fig. 4a). Additional Pdc expression can increase ethanol production $^{39}$. The pTy1-Pd plasmid possesses the PDC1 gene, a major gene among the PDC gene family, with the Psyn promoter and Tguo1 terminator and the LEU2 marker gene. Cells with the pTy1-Pd plasmid were cultured in SC-Leu $(-\mathrm{L})$ and SC $(+\mathrm{L})$ for $24 \mathrm{~h}$ and with wild-type (BY4742) cells in SC. The amount of ethanol released into the medium by cells with the pTy1-Pd plasmid $(-\mathrm{L})$ increased more than wild-type cells, although the ethanol level in cells with the pTy1-Pd plasmid $(+\mathrm{L})$ was almost similar to that in wild-type cells (Fig. $4 \mathrm{~b})$. These data demonstrate that the CRITGI system can produce the metabolite of interest by using amino acids.

In this research, we have developed a novel gene expression system named CRITGI. CRITGI can control gene expression in the pTy1 plasmid integrated into Ty1 by using marker gene activation. For maintaining plasmid in budding yeast, we usually use genes involved in amino acid syntheses and nucleotide synthesis as plasmid markers ${ }^{18}$. Together with conventional molecules that drive inducible promoters, for example, galactose for the GAL1/10 promoter $^{40}$, cupper for the CUP1 promoter ${ }^{41}$ and tetracycline for the tet promoter $^{42}$, various amino acids or nucleotides are available to control gene expression by CRITGI, as long as the genes involved in their syntheses are applicable for the pTy1 plasmid marker genes. CRITGI uses CRISPR/Cas9 to integrate multiple copies of a plasmid into Ty1 loci. The integration number of a plasmid is proportional to the protein expression level. These advantages of CRITGI contribute to metabolic engineering. Fine-tuning the timing and levels of the expression of enzymes involved in both natural and engineered pathways can relieve bottlenecks and minimize the 
metabolic burden of chemical production $39,43,44$. The production of an end product requires the complete set of metabolic pathways of interest. The incomplete set of the metabolic pathway of interest, which lacks any enzyme steps, might cause the abnormal accumulation of metabolic intermediate(s) harmful for cell survival. CRITGI can induce multiple enzyme-encoding genes that are composed of the metabolic pathway and control the expression levels of enzymes. Although further development of the CRITGI system is needed in the future, CRITGI, which can express a set of enzymes of a metabolic pathway of interest, will contribute to industries such as metabolic engineering, materials engineering, and pharmaceuticals.

Native promoters in Saccharomyces cerevisiae regulated by carbon sources ${ }^{45,46}$ or the availability of specific nutrients ${ }^{47,48}$ are applicable to express or repress genes of interest in a trans-acting factor-dependent manner. CRITGI has a unique gene induction system; marker gene activation evokes Tyl transcription, which is necessary to induce a gene on PTY1plasmid. We favor that Ty1 transcription alters the chromatin structure favorable for transcription of a gene from the pTy1 plasmid. (1) Genes on the pTy1 plasmid need their own promoters. (2) MNase-ChIP shows that $\mathrm{H} 3-\mathrm{K} 4 \mathrm{me} 3$ accumulates at $5^{\prime}$ end of a gene in $\mathrm{pTy} 1$ following marker gene activation. (3) Two genes that are set in tandem on the pTyl plasmid can be synchronously expressed. Swi/Snf and ISWI chromatin remodeling complexes are involved in Ty1 mRNA transcription from the LTR together with transcriptional factors, such as $\mathrm{Gcn} 5^{20,33}$, suggesting that chromatin remodeling factors may create a chromatin structure favorable for gene transcription from the pTyl-gene plasmid. CRITGI is a unique gene regulation system that alters the Tyl chromatin structure from repressed to competent allowing gene transcription. Further efforts need to reveal the mechanism to create a chromatin environment favorable for gene transcription within the Tyl element in the future.

\section{Methods}

Strains and media. The genotypes of the strains, plasmids and primers used in this study are listed in Table S1. The parental budding yeast strain used in the present study was BY4742 (MAT $\alpha$ his $3 \Delta$ leu2 $\Delta 1$ met $15 \Delta 0$ ura $3 \Delta 0)^{49}$. A yeast strain harboring a single gene deletion was commercially available from the haploid yeast open reading frame deletion collection ${ }^{50}$ (GE Dharmacon, Lafayette, CO, USA). Yeast cells were routinely grown at $30^{\circ} \mathrm{C}$ in YPD ( $1 \%$ yeast extract, $2 \%$ peptone, $2 \%$ glucose $)$ or appropriate synthetic complete (SC) medium ${ }^{51,52}$. If necessary, the media were solidified by including $2 \%$ agar. For the time course analysis, yeast cells were cultured at $25^{\circ} \mathrm{C}$. A standard method was used for isolation of the yeast genome DNA ${ }^{52}$. E. coli strain DH5 $\alpha^{53}$, and standard media and methods were used for plasmid manipulations ${ }^{54}$. For the isolation of plasmid free of dam methylation, INV110 E. coli competent cells (Thermo Fisher Scientific, Waltham, MA, USA) were used.

Plasmid construction. To construct plasmid PHM661 (pTy1), the $\sim 870$ bp PCR product (Ty1 homologous recombination (HR) sequence, ranging from 1681 bp to 2541 bp in YPRWTy1-3) obtained using BY4742 genomic DNA as a template, with forward primer (HMP1196) and reverse primer (HMP1197), was digested with Bam $\mathrm{HI}$ and Hind III and ligated into Bam HI/Hind III-digested YIplac128 plasmid ${ }^{55}$. For the construction of plasmid PHM688, the Bam HI/Hind III-digested Ty1 PCR fragment was ligated into the Bam HI/Hind III-digested pRS403 plasmid ${ }^{56}$.

To construct the plasmid encoding Cas9 and crRNA, we used pML104 plasmid (gift from John Wyrick (Addgene plasmid \# 67638)) as a base plasmid ${ }^{57}$. This plasmid requires the insertion of the 20 -nt guide sequence within a single guide RNA (sgRNA) cassette. The construction of the sgRNA cassette in the pML104 plasmid was described elsewhere ${ }^{6,57,58}$. The target sequence was set within the Ty1 HR sequence for the guide RNA (gTy1 \#3) (5'-ACGTCTTAGAACGGTCTGACGG-3' (underline: PAM sequence)) and set within the short Tyl HR sequence for gTy1 \#4 (5'-ACCTACATACTGACATATTTGG-3'). To construct the 20-bp guide sequence within the sgRNA, two DNA primers (HMP1192 and HMP1193 for gTy1 \#3; HMP1194 and HMP1195 for gTy1 \#4) were mixed at a final concentration of $10 \mu \mathrm{M}$ in annealing buffer $\left(40 \mathrm{mM}\right.$ Tris-Cl pH 8.0, $20 \mathrm{mM} \mathrm{MgCl}_{2}$ and $50 \mathrm{mM} \mathrm{NaCl}$ ). The mixture was incubated at $95^{\circ} \mathrm{C}$ for $5 \mathrm{~min}$ and cooled down for $90 \mathrm{~min}$ until it reached $25^{\circ} \mathrm{C}$. To obtain pML104 plasmid free of dam methylation because Dam methylation blocks $B c l$ I digestion, the pML104 plasmid was transformed into a dam- E. coli competent cell (INV110). To construct the PHM663 (gTy1 \#3) and PHM664 (gTy1 \#4) plasmids, 20-bp double strand DNA cassettes (gTy1 \#3 and gTY1 \#4) were ligated into Bcl $\mathrm{I} / \mathrm{Swa}$ I-digested pML104 plasmid. The ligation mixture $(2.5 \mu \mathrm{l}$ of $25 \mathrm{ng} / \mu \mathrm{l}$ pML104, $0.5 \mu \mathrm{l}$ of the annealed DNA strand, $0.1 \mu \mathrm{l}$ of $10 \mathrm{units} / \mu \mathrm{l} \mathrm{Bcl} \mathrm{I,} 0.1 \mu \mathrm{l}$ of 10 units/ $\mu \mathrm{l}$ Swa I, $0.1 \mu \mathrm{l}$ of Quick ligase, $1 \mathrm{x}$ T4 DNA ligase buffer (New England Biolabs, Ipswich, MA)) was run for 3 cycles of $5 \mathrm{~min}$ at $37^{\circ} \mathrm{C}, 10 \mathrm{~min}$ at $16^{\circ} \mathrm{C}$. Two $\mu \mathrm{l}$ of the ligation mixture was transformed into DH5 $\alpha$ E. coli competent cells to obtain the plasmids (PHM663 and PHM664). Construction of other plasmids is described in Supplementary method.

Yeast transformation for CRITGI. Yeast transformation was described elsewhere ${ }^{59,60}$. Yeast cells were grown on YPD solid plates, collected with toothpicks and suspended in $100 \mu$ of one-step buffer containing a plasmid mixture ${ }^{59}$. The mixture with $0.5 \sim 1.0 \mu \mathrm{g}$ of integration plasmid (LEU2 or HIS3 marker gene) and $0.5 \sim 1.0 \mu \mathrm{g}$ of Cas9/gRNA plasmid (URA3 marker) was used for transformation. The cell suspension was incubated at $42^{\circ} \mathrm{C}$ for $1 \mathrm{~h}$ and then plated on SC uracil and either leucine or histidine dropout medium (SC-Ura-Leu or SC-Ura-His). The transformants, plasmids, and parent strains are listed in Table S1. We routinely used the HMY1448 and HMY1459 strains, which have multiple copies of the PHM661 (LEU2) and PHM668 (HIS3) plasmids already integrated in Tyl loci, respectively. These strains are useful in obtaining transformants harboring multiple copies of plasmids by transformation.

The isolation of chromosomal DNA from yeast transformants was described elsewhere ${ }^{52}$. To measure the number of plasmids integrated into Ty1 loci, we used the LightCycler 480 SYBR Green I Master (Roche Life Science, Penzberg, Germany). The mix contained $10 \mu$ of $2 x$ LightCycler 480 SYBR Green I Master Mix, $2 \mu \mathrm{l}$ of $5 \mu \mathrm{M}$ PCR forward primer, $2 \mu \mathrm{l}$ of $5 \mu \mathrm{M}$ PCR reverse primer and $\sim 10 \mathrm{ng}$ of total DNA (final volume $20 \mu \mathrm{l}$ ). 
Reactions were run for 1 cycle of $10 \mathrm{~min}$ at $95^{\circ} \mathrm{C} ; 45$ cycles of $20 \mathrm{sec}$ at $95^{\circ} \mathrm{C}, 20 \mathrm{sec}$ at $53^{\circ} \mathrm{C}$ and $20 \sec$ at $72^{\circ} \mathrm{C} ; 1$ cycle of $60 \mathrm{sec}$ at $65^{\circ} \mathrm{C}$ and $1 \mathrm{sec}$ at $95^{\circ} \mathrm{C}$ using either a LightCycler 480 System II or LightCycler Nano (Roche Life Science, Penzberg, Germany). To calculate the plasmid integration number, the LEU2 gene and HIS3 gene on the plasmid were compared to the ACT1 gene as one copy. PCR primers are listed in Table S1.

Ethanol quantification assay. The cells were cultured overnight in YPD medium at $25^{\circ} \mathrm{C}$. Cells $\left(1 \times 10^{7}\right.$ cells $/ \mathrm{ml}$ ) were resuspended in SC-Leu or SC ( + Leu) media and then cultured at $25^{\circ} \mathrm{C}$ with shaking for $24 \mathrm{~h}$. An aliquot $(1 \mathrm{ml})$ of the culture was sampled, and the medium and cell pellet were separated by centrifugation. The medium was transferred to a new Eppendorf tube and heated for $5 \mathrm{~min}$ at $70^{\circ} \mathrm{C}$ to inactivate the enzymes that consume ethanol in the medium. The heat-treated medium $(10 \mu \mathrm{l})$ was assayed using the Ethanol Colorimetric/ Fluorometric Assay Kit (BioVision, Milpitas, CA, USA) according to the manufacturer's instructions. Fluorescence changes $(\mathrm{Ex} / \mathrm{Em}=535 / 587 \mathrm{~nm})$ were monitored using an Infinite F200 fluorescence microplate reader (TECAN, Männedorf, Switzerland). At least three replicates were analyzed for each strain.

RNA isolation and real time (RT)-PCR. Total RNA was isolated from budding yeast using the RNeasy Mini Kit (Qiagen, Santa Clarita, CA, USA). The relative comparison of the mRNA amount was performed using a One Step SYBR PrimeScript PLUS RT-PCR Kit (Takara-Bio, Kusatsu, Shiga, Japan). The mix contained $10 \mu \mathrm{l}$ of 2x One Step SYBR RT-PCR Buffer 4, $1.2 \mu \mathrm{l}$ of Takara Ex Taq HS Mix, $0.4 \mu \mathrm{l}$ of PrimeScript PLUS RTase Mix, $0.8 \mu \mathrm{l}$ of $10 \mu \mathrm{M}$ PCR forward primer, $0.8 \mu \mathrm{l}$ of $10 \mu \mathrm{M}$ PCR reverse primer and $100 \mathrm{ng}$ of total RNA (final volume $20 \mu \mathrm{l}$ ). Reactions were run for $1 \mathrm{cycle}$ of $5 \mathrm{~min}$ at $42^{\circ} \mathrm{C} ; 1 \mathrm{cycle}$ of $10 \mathrm{sec}$ at $95^{\circ} \mathrm{C} ; 40 \mathrm{cycles}$ of $5 \mathrm{sec}$ at $95^{\circ} \mathrm{C}, 20 \mathrm{sec}$ at $55^{\circ} \mathrm{C}$; $1 \mathrm{cycle}$ of $1 \mathrm{sec}$ at $95^{\circ} \mathrm{C} ; 1 \mathrm{cycle}$ of $15 \mathrm{sec}$ at $65^{\circ} \mathrm{C}$ and $1 \mathrm{sec}$ at $95^{\circ} \mathrm{C}$ using either a LightCycler 480 System II or LightCycler Nano (Roche Life Science, Penzberg, Germany). The amount of each mRNA was compared with the amount of ACT1 mRNA. PCR primers are listed in Table S1.

Western blotting. Yeast cells expressing FLAG-tagged protein were used. The protein extraction was described elsewhere ${ }^{61}$. Proteins were separated by $15 \%$ sodium dodecyl sulfate-polyacrylamide gel electrophoresis (SDS-PAGE) and transferred to nitrocellulose membranes (Amersham Protran) (GE healthcare, Little Chalfont, Buckinghamshire, England). The protein level in each SDS-PAGE lane was normalized and then confirmed using 0.1\% Ponceau S solution (Sigma-Aldrich, St. Louis, MO, USA). Mouse monoclonal anti-FLAG M2 antibody (1:1000 dilution) (Sigma-Aldrich, St. Louis, MO, USA) was used to detect FLAG-tagged protein.

Micrococcus nuclease-chromatin immunoprecipitation (MNase-ChIP) assay. HMY1494 cells (4 copies of PHM820 plasmid) were cultured overnight in $20 \mathrm{ml}$ of YPD medium at $25^{\circ} \mathrm{C}$. Cells ( $3 \mathrm{ml}$ ) were grown either in SC-Leu or SC media shaking at $25^{\circ} \mathrm{C}$ for $5 \mathrm{~h}$ (OD600: 0.7 0.9). Cells were fixed with formaldehyde at a final concentration of $1 \%$ for $15 \mathrm{~min}$. Cell pellets were harvested, washed with TBS buffer $(20 \mathrm{mM}$ Tris-Cl $\mathrm{pH} 7.5,150 \mathrm{mM} \mathrm{NaCl}$ ), and conserved at $-20^{\circ} \mathrm{C}$ until use. The cell pellets were subjected to zymolase treatment to make spheroplast, MNase digestion, and chromatin immunoprecipitation ${ }^{35,36}$. Monoclonal antibody to recognize trimethylated histone H3 on lysine 4 (H3-K4 me3) (04-745, Merk Millipore, Burlington, MA, USA) was employed for ChIP ${ }^{36}$. The LightCycler 480 SYBR Green I Master (Roche Life Science, Penzberg, Germany) was used to calculate the percent of input for ChIP for histone H3-K4 me3. The mix contained $10 \mu \mathrm{l}$ of $2 \mathrm{x}$ LightCycler 480 SYBR Green I Master, $2 \mu \mathrm{l}$ of $5 \mu \mathrm{M}$ PCR forward primer, $2 \mu \mathrm{l}$ of $5 \mu \mathrm{M}$ PCR reverse primer and $\sim 10 \mathrm{ng}$ of total DNA (final volume $20 \mu \mathrm{l}$ ). Reactions were run for $1 \mathrm{cycle}$ of $10 \mathrm{~min}$ at $95^{\circ} \mathrm{C} ; 45 \mathrm{cycles}$ of $20 \mathrm{sec}$ at $95^{\circ} \mathrm{C}, 20 \mathrm{sec}$ at $53^{\circ} \mathrm{C}$, and $20 \mathrm{sec}$ at $72^{\circ} \mathrm{C} ; 1 \mathrm{cycle}$ of $60 \mathrm{sec}$ at $65^{\circ} \mathrm{C}$ and $1 \mathrm{sec}$ at $95^{\circ} \mathrm{C}$ using LightCycler Nano (Roche Life Science, Penzberg, Germany). The Ty1 500, Flag-Venus (N) and ACT1 regions were used to monitor the status of histone H3-K4 me3. PCR primers are listed in Table S1. The ratio of the percent of input for ChIP in the SC-Leu culture over the SC culture was calculated.

Received: 21 August 2018; Accepted: 9 October 2019;

Published online: 25 October 2019

\section{References}

1. Sorek, R., Lawrence, C. M. \& Wiedenheft, B. CRISPR-mediated adaptive immune systems in bacteria and archaea. Annu Rev Biochem 82, 237-266, https://doi.org/10.1146/annurev-biochem-072911-172315 (2013).

2. Doudna, J. A. \& Charpentier, E. Genome editing. The new frontier of genome engineering with CRISPR-Cas9. Science 346, 1258096, https://doi.org/10.1126/science.1258096 (2014).

3. Hsu, P. D., Lander, E. S. \& Zhang, F. Development and applications of CRISPR-Cas9 for genome engineering. Cell 157, 1262-1278, https://doi.org/10.1016/j.cell.2014.05.010 (2014).

4. Mohanraju, P. et al. Diverse evolutionary roots and mechanistic variations of the CRISPR-Cas systems. Science 353, aad5147, https:// doi.org/10.1126/science.aad5147 (2016)

5. Jinek, M. et al. RNA-programmed genome editing in human cells. Elife 2, e00471, https://doi.org/10.7554/eLife.00471 (2013).

6. Ran, F. A. et al. Genome engineering using the CRISPR-Cas9 system. Nat Protoc 8, 2281-2308, https://doi.org/10.1038/ nprot.2013.143 (2013).

7. Hwang, W. Y. et al. Efficient genome editing in zebrafish using a CRISPR-Cas system. Nat Biotechnol 31, 227-229, https://doi. org/10.1038/nbt.2501 (2013).

8. Friedland, A. E. et al. Heritable genome editing in C. elegans via a CRISPR-Cas9 system. Nat Methods 10, 741-743, https://doi. org/10.1038/nmeth.2532 (2013).

9. Gratz, S. J. et al. Genome engineering of Drosophila with the CRISPR RNA-guided Cas9 nuclease. Genetics 194, 1029-1035, https:// doi.org/10.1534/genetics.113.152710 (2013).

10. Cho, S. W., Kim, S., Kim, J. M. \& Kim, J. S. Targeted genome engineering in human cells with the Cas9 RNA-guided endonuclease. Nat Biotechnol 31, 230-232, https://doi.org/10.1038/nbt.2507 (2013). 
11. Mao, Y. et al. Application of the CRISPR-Cas system for efficient genome engineering in plants. Mol Plant 6, 2008-2011, https://doi. org/10.1093/mp/sst121 (2013).

12. Shi, S., Liang, Y., Zhang, M. M., Ang, E. L. \& Zhao, H. A highly efficient single-step, markerless strategy for multi-copy chromosomal integration of large biochemical pathways in Saccharomyces cerevisiae. Metab Eng 33, 19-27, https://doi.org/10.1016/j. ymben.2015.10.011 (2016).

13. Sasano, Y., Nagasawa, K., Kaboli, S., Sugiyama, M. \& Harashima, S. CRISPR-PCS: a powerful new approach to inducing multiple chromosome splitting in Saccharomyces cerevisiae. Sci Rep 6, 30278, https://doi.org/10.1038/srep30278 (2016).

14. Gilbert, L. A. et al. CRISPR-mediated modular RNA-guided regulation of transcription in eukaryotes. Cell 154, 442-451, https://doi. org/10.1016/j.cell.2013.06.044 (2013).

15. Tsarmpopoulos, I. et al. In-Yeast Engineering of a Bacterial Genome Using CRISPR/Cas9. ACS Synth Biol 5, 104-109, https://doi. org/10.1021/acssynbio.5b00196 (2016).

16. Si, T. et al. Automated multiplex genome-scale engineering in yeast. Nat Commun 8, 15187, https://doi.org/10.1038/ncomms15187 (2017).

17. DiCarlo, J. E., Chavez, A., Dietz, S. L., Esvelt, K. M. \& Church, G. M. Safeguarding CRISPR-Cas9 gene drives in yeast. Nat Biotechnol 33, 1250-1255, https://doi.org/10.1038/nbt.3412 (2015).

18. Dunham, M. J. G. \& M. R. Brown, G. W. Methods in Yeast Genetics and Genomics. 2015 Edition edn, (Cold Spring Harbor Laboratory Press, 2015).

19. Kim, J. M., Vanguri, S., Boeke, J. D., Gabriel, A. \& Voytas, D. F. Transposable elements and genome organization: a comprehensive survey of retrotransposons revealed by the complete Saccharomyces cerevisiae genome sequence. Genome Res 8, 464-478 (1998).

20. Curcio, M. J., Lutz, S. \& Lesage, P. The Ty1 LTR-retrotransposon of budding yeast, Saccharomyces cerevisiae. Microbiol Spectr 3, 1-35, https://doi.org/10.1128/microbiolspec.MDNA3-0053-2014 (2015).

21. Mieczkowski, P. A., Lemoine, F. J. \& Petes, T. D. Recombination between retrotransposons as a source of chromosome rearrangements in the yeast Saccharomyces cerevisiae. DNA Repair (Amst) 5, 1010-1020, https://doi.org/10.1016/j. dnarep.2006.05.027 (2006).

22. Jorgensen, R. A. Cosuppression, flower color patterns, and metastable gene expression States. Science 268, 686-691, https://doi. org/10.1126/science.268.5211.686 (1995).

23. Jiang, Y. W. Transcriptional cosuppression of yeast Ty1 retrotransposons. Genes Dev 16, 467-478, https://doi.org/10.1101/ gad.923502 (2002).

24. Mumberg, D., Muller, R. \& Funk, M. Yeast vectors for the controlled expression of heterologous proteins in different genetic backgrounds. Gene 156, 119-122 (1995).

25. Nagai, T. et al. A variant of yellow fluorescent protein with fast and efficient maturation for cell-biological applications. Nat Biotechnol 20, 87-90, https://doi.org/10.1038/nbt0102-87 (2002).

26. Redden, H. \& Alper, H. S. The development and characterization of synthetic minimal yeast promoters. Nat Commun 6, 7810, https://doi.org/10.1038/ncomms8810 (2015).

27. Curran, K. A. et al. Short Synthetic Terminators for Improved Heterologous Gene Expression in Yeast. ACS Synth Biol 4, 824-832, https://doi.org/10.1021/sb5003357 (2015).

28. Hsu, S. T. et al. Folding study of Venus reveals a strong ion dependence of its yellow fluorescence under mildly acidic conditions. J Biol Chem 285, 4859-4869, https://doi.org/10.1074/jbc.M109.000695 (2010).

29. Grant, P. A. et al. Yeast Gcn5 functions in two multisubunit complexes to acetylate nucleosomal histones: characterization of an Ada complex and the SAGA (Spt/Ada) complex. Genes Dev 11, 1640-1650 (1997).

30. Sudarsanam, P., Cao, Y., Wu, L., Laurent, B. C. \& Winston, F. The nucleosome remodeling complex, Snf/Swi, is required for the maintenance of transcription in vivo and is partially redundant with the histone acetyltransferase, Gcn5. EMBO J 18, 3101-3106, https://doi.org/10.1093/emboj/18.11.3101 (1999).

31. Peterson, C. L. \& Workman, J. L. Promoter targeting and chromatin remodeling by the SWI/SNF complex. Curr Opin Genet Dev 10, $187-192(2000)$

32. Kent, N. A., Karabetsou, N., Politis, P. K. \& Mellor, J. In vivo chromatin remodeling by yeast ISWI homologs Isw1p and Isw2p. Genes Dev 15, 619-626, https://doi.org/10.1101/gad.190301 (2001).

33. Servant, G., Pennetier, C. \& Lesage, P. Remodeling yeast gene transcription by activating the Ty1 long terminal repeat retrotransposon under severe adenine deficiency. Mol Cell Biol 28, 5543-5554, https://doi.org/10.1128/MCB.00416-08 (2008).

34. Morillon, A., Benard, L., Springer, M. \& Lesage, P. Differential effects of chromatin and Gcn4 on the 50-fold range of expression among individual yeast Ty1 retrotransposons. Mol Cell Biol 22, 2078-2088 (2002)

35. Liu, C. L. et al. Single-nucleosome mapping of histone modifications in S. cerevisiae. PLoS Biol 3, e328, https://doi.org/10.1371/ journal.pbio.0030328 (2005).

36. Weiner, A. et al. High-resolution chromatin dynamics during a yeast stress response. Mol Cell 58, 371-386, https://doi.org/10.1016/j. molcel.2015.02.002 (2015).

37. Masumoto, H., Hawke, D., Kobayashi, R. \& Verreault, A. A role for cell-cycle-regulated histone H3 lysine 56 acetylation in the DNA damage response. Nature 436, 294-298, https://doi.org/10.1038/nature03714 (2005).

38. Belle, A., Tanay, A., Bitincka, L., Shamir, R. \& O'Shea, E. K. Quantification of protein half-lives in the budding yeast proteome. Proc Natl Acad Sci USA 103, 13004-13009, https://doi.org/10.1073/pnas.0605420103 (2006).

39. Zhao, E. M. et al. Optogenetic regulation of engineered cellular metabolism for microbial chemical production. Nature 555, 683-687, https://doi.org/10.1038/nature26141 (2018).

40. Guarente, L. Yeast promoters: positive and negative elements. Cell 36, 799-800, https://doi.org/10.1016/0092-8674(84)90028-x (1984).

41. Etcheverry, T. Induced expression using yeast copper metallothionein promoter. Methods Enzymol 185, 319-329, https://doi. org/10.1016/0076-6879(90)85028-m (1990).

42. Gossen, M. et al. Transcriptional activation by tetracyclines in mammalian cells. Science 268, 1766-1769, https://doi.org/10.1126/ science.7792603 (1995).

43. Keasling, J. D. Manufacturing molecules through metabolic engineering. Science 330, 1355-1358, https://doi.org/10.1126/ science.1193990 (2010).

44. Ajikumar, P. K. et al. Isoprenoid pathway optimization for Taxol precursor overproduction in Escherichia coli. Science 330, 70-74, https://doi.org/10.1126/science.1191652 (2010).

45. Da Silva, N. A. \& Srikrishnan, S. Introduction and expression of genes for metabolic engineering applications in Saccharomyces cerevisiae. FEMS Yeast Res 12, 197-214, https://doi.org/10.1111/j.1567-1364.2011.00769.x (2012).

46. Lee, K. M. \& DaSilva, N. A. Evaluation of the Saccharomyces cerevisiae ADH2 promoter for protein synthesis. Yeast 22, 431-440, https://doi.org/10.1002/yea.1221 (2005)

47. Ro, D. K. et al. Production of the antimalarial drug precursor artemisinic acid in engineered yeast. Nature 440, 940-943, https://doi. org/10.1038/nature04640 (2006).

48. Mao, X., Hu, Y., Liang, C. \& Lu, C. MET3 promoter: a tightly regulated promoter and its application in construction of conditional lethal strain. Curr Microbiol 45, 37-40, https://doi.org/10.1007/s00284-001-0046-0 (2002). 
49. Brachmann, C. B. et al. Designer deletion strains derived from Saccharomyces cerevisiae S288C: a useful set of strains and plasmids for PCR-mediated gene disruption and other applications. Yeast 14, 115-132, doi:10.1002/(SICI) 1097-0061(19980130) 14:2<115::AID-YEA204>3.0.CO;2-2 (1998).

50. Winzeler, E. A. et al. Functional characterization of the S. cerevisiae genome by gene deletion and parallel analysis. Science $\mathbf{2 8 5}$, 901-906 (1999).

51. Sherman, F. Getting started with yeast. Methods Enzymol 350, 3-41 (2002).

52. Amberg, D. C., Burke, F. J. \& Strathern, J. N. Methods in Yeast Genetics 2005 Edition. (Cold Spring Harbor Laboratory Press, 2005).

53. Taylor, R. G., Walker, D. C. \& McInnes, R. R. E. coli host strains significantly affect the quality of small scale plasmid DNA preparations used for sequencing. Nucleic Acids Res 21, 1677-1678 (1993).

54. Ausubel, F. M. et al. Current Protocols in Molecular Biology. (John Wiley \& Sons, New York, 1995).

55. Gietz, R. D. \& Sugino, A. New yeast-Escherichia coli shuttle vectors constructed with in vitro mutagenized yeast genes lacking sixbase pair restriction sites. Gene 74, 527-534 (1988).

56. Sikorski, R. S. \& Hieter, P. A system of shuttle vectors and yeast host strains designed for efficient manipulation of DNA in Saccharomyces cerevisiae. Genetics 122, 19-27 (1989).

57. Laughery, M. F. et al. New vectors for simple and streamlined CRISPR-Cas9 genome editing in Saccharomyces cerevisiae. Yeast 32, 711-720, https://doi.org/10.1002/yea.3098 (2015).

58. Mashiko, D. et al. Generation of mutant mice by pronuclear injection of circular plasmid expressing Cas 9 and single guided RNA. Sci Rep 3, 3355, https://doi.org/10.1038/srep03355 (2013).

59. Chen, D. C., Yang, B. C. \& Kuo, T. T. One-step transformation of yeast in stationary phase. Curr Genet 21, 83-84 (1992).

60. Akada, R., Kawahata, M. \& Nishizawa, Y. Elevated temperature greatly improves transformation of fresh and frozen competent cells in yeast. Biotechniques 28, 854-856 (2000).

61. Kushnirov, V. V. Rapid and reliable protein extraction from yeast. Yeast 16, 857-860, doi:10.1002/1097-0061(20000630) 16:9<857::AID-YEA561 >3.0.CO;2-B (2000).

\section{Author contributions}

M.H. and H.M. performed the experiments and prepared the figures. H.M. wrote the manuscript text. All authors reviewed the manuscript.

\section{Competing interests}

The authors declare no competing interests.

\section{Additional information}

Supplementary information is available for this paper at https://doi.org/10.1038/s41598-019-51891-6.

Correspondence and requests for materials should be addressed to H.M.

Reprints and permissions information is available at www.nature.com/reprints.

Publisher's note Springer Nature remains neutral with regard to jurisdictional claims in published maps and institutional affiliations.

Open Access This article is licensed under a Creative Commons Attribution 4.0 International License, which permits use, sharing, adaptation, distribution and reproduction in any medium or format, as long as you give appropriate credit to the original author(s) and the source, provide a link to the Creative Commons license, and indicate if changes were made. The images or other third party material in this article are included in the article's Creative Commons license, unless indicated otherwise in a credit line to the material. If material is not included in the article's Creative Commons license and your intended use is not permitted by statutory regulation or exceeds the permitted use, you will need to obtain permission directly from the copyright holder. To view a copy of this license, visit http://creativecommons.org/licenses/by/4.0/.

(C) The Author(s) 2019 